\title{
Real Time Moving Object Detection
}

\author{
Mr.Yogesh J Chaudhari ${ }^{1}$, Mr.Aman R. Shaikh ${ }^{2}$, Mr.Rushikesh S. Londhe', \\ Mr.Rohan M. Saggam ${ }^{4}$, Prof. Rakhi Bharadwaj ${ }^{5}$ \\ Student, Comp Dept, KJEI's Trinity college, Pune, India ${ }^{1,2,3,4}$ \\ Guide, Comp Dept, KJEI's Trinity college, Pune, India ${ }^{5}$
}

\begin{abstract}
In real world application, video security is becoming more important now-a-days due to the happening of unwanted events in our surroundings. Moving object detection is a challenging task in low resolution video, variable lightening conditions and in crowed area due to the limitation of pattern recognition techniques and it looses many important details in the visual appearance of the moving object.[1]Video surveillance system is a process of monitoring and analysing video sequences for the purpose of checking the behaviour, activities and other certain information in a video sequence. It is really a very upcoming area in the real time system. It takes lot of data to storage in computer system. In Manual video surveillance the video contents are analysed by human being and this type of Manual systems are mostly used in real time system. But Semi- automated video surveillance system consists of both human intervention and up to some extent of video processing. The function of the video capture section is to take input video data from the individual cameras connected in the local area network and each of the cameras is accessed through the different IP address. And captured video is correct and failure in the video.
\end{abstract}

Keywords: Semi- Automated Video Surveillance, K-means Algorithm, Object Detecting, Image Analysis, Image Identification, Image Understanding

\section{INTRODUCTION}

Video surveillance system is a process to analysing video sequences for the purpose of checking the behaviour, activities and other certain information in a video sequence. It is really a very upcoming area in the real time system. It takes lot of data to storage in computer system. In Manual video surveillance the video contents are analyzed by human being and this type of Manual systems are mostly used in real time system. Most useful examples of this type of system are found in simple moving object detection in video sequence. A fully autonomous system is used for extra ordinary motion in captured video and then sent to the human expert for further analysis. In case of fully-autonomous system, video sequence is the input which is taken from the source where we had performed the surveillance system. So, this part basically tells whether the captured video is corrected or any failure in the video capture process.

\section{LITERATURE SURVEY}

Title: Analysis of Moving Object Detection and Tracking in Video Surveillance System.

Author: Kamal Kant Verma, Pradeep Kumar, AnkitTomar

Moving object detection is a challenging task in low resolution video, variable lightening conditions and in crowed area due to the limitation of pattern recognition techniques and it loses many important details in the visual appearance of the moving object. In this paper we propose a review on unusual event detection in video surveillance system. Video surveillance system might be used for enhancing the security in various organizations, academic institutions and many more areas.

Title: A Design of Detection and Tracing System Based on Dynamic Multi-Object

Author: Wantao Kou1, Yu Wang2, Jinxu Guo2, Hao Zhang, Shengfa Liu1

The captured image is divided into the foreground and background by the mixed Gauss background model, and then the dynamic target of each frame in the video is found. The Camshift algorithm based on Kalman filter and feature fusion is used to track it. In this process, real time video camera is used to obtain the real-time video. The experimental results show that the Kalman filter can well predict the target's position to reduce the number of iterations in the Camshift algorithm, the texture feature and color feature selection combining histogram can track the target better, reduce loss rate, improve the real-time target detection and tracking.

Title: Detection of a Specific Moving Object from Head-mounted Camera Images Author: Katsuma Ishitobi, Joo Kooi Tan 
A method is proposed for detecting and tracking a specific moving object (e.g., a bus) on the road From these optical flows, we remove the optical flows of camera motion using projective transformation and RANSAC from images of a camera attached to the head of a user, aiming. A moving object region is estimated from the remaining at developing a system to support daily lives of visually impaired optical flows. Haar-like feature [9] and random trees [10] are people. The proposed method traces feature points on the applied to this estimated moving object region to find a images, extracts a moving object region, and detects a bus by applying Haar-like feature and random trees to the region. The effectiveness of the proposed method is shown experimentally.

Title: A Stereo Cameras Setup for Pedestrian Detection Enhancement

Author : Angelica Tiemi Mizuno Nakamura, Luiz Ricardo Takeshi Horita, Valdir Grassi .

Pedestrian detection is an important and challenging area in computer vision with the potential to save lives. Although many works have already been developed to improve performance of pedestrian detection algorithms, none have yet been able to handle detection from afar due to pedestrians' small scales on images. This prevents these algorithms to be reliably applied to autonomous vehicles to avoid accidents on fast moving traffic. In this context, this paper proposes a cameras setup, which consists of two stereo cameras with different focal lengths and baselines, allowing to have higher pedestrian resolutions on images for a larger range of distances in front of the vehicle. Experimental results reveal a considerable enhancement on the detection performance, overcoming the difficulty caused by the reduced scale that pedestrians have on images.

\section{PROPOSED SYSTEM}

In Proposed system we are analysing the real time moving object. The camera can capture the video. And Detect the moving object. Using $\mathrm{K}$ means algorithm some operation can be perform. The operation like Image Analysis, Image understanding, Image identification. User can captured the video and it is converted into image Then this image on applied $\mathrm{k}$-means algorithm. Then image are Analysis and then this image will be understand. After understanding image are identified and display the moving object . it will also used in environments like detect the car, person and vehicle.

\section{SYSTEM ARCHITECTURE}

Following diagram is our system's architecture diagram:

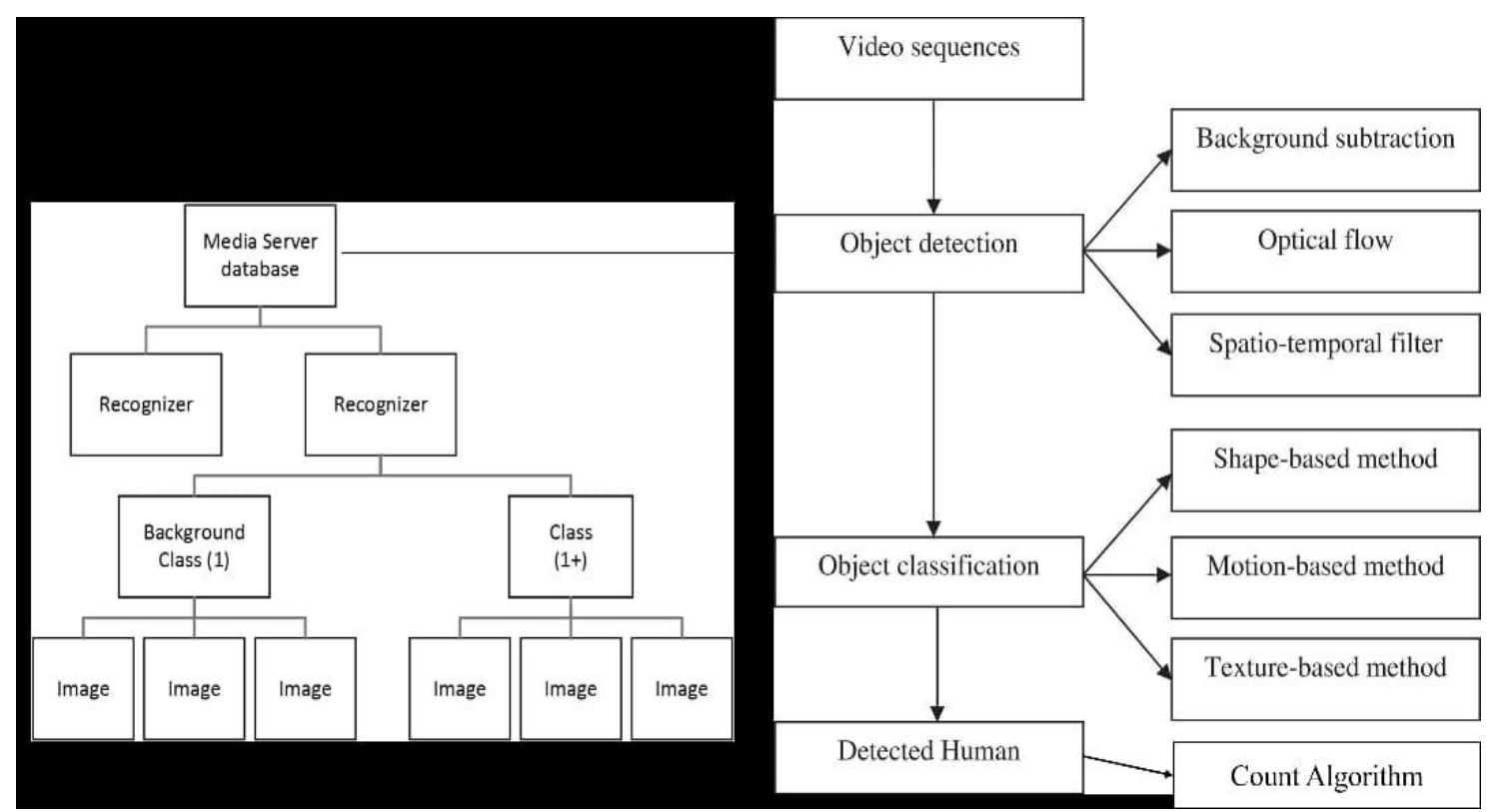

Figure 1: system architecture

In system architecture camera capture the video and then moving object are detected. Then using K-means algorithm image are processed in three steps. This steps are Image Analysis , Image understanding and Image Identification. And lastly result are displayed. 


\title{
IJARCCE
}

\section{International Journal of Advanced Research in Computer and Communication Engineering}

\author{
Vol. 7, Issue 11, November 2018
}

\section{METHODOLOGIES}

We used mythologies in our project like object detecting, image analysis, image identification, image understanding

1.Image detecting :- user can capture the video and video is converted into images.

2.Image analysis :- The video is converted into image then using $\mathrm{K}$ means algorithm image is analyzed.

3.Image understanding :- After image Analysis image are understood. It means it will detect image quality .

4.Image identification.$:$ - After all the image process is complete then image can be identified. And find moving object in that video.

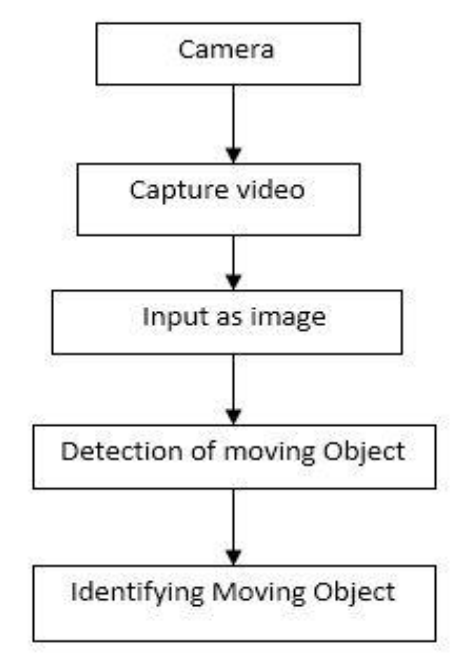

Figure 2:Data flow diagram

\section{CONCLUSION}

The proposed system will try to increase the accuracy of detection of the object using deep learning and keep the count of the persons detected in video. And also in Environment the moving object like a Car, vehicle it will also detect.

\section{REFERRENCES}

[1]. W.Lizhe, Jie Tao, M.Kunze, A.C. Castellanos, D.Kramer, and W.Karl,"Scientific Cloud Computing: Early Definition and Experience," In HPCC, vol. 8, pp. 825-830. Sep 2008.

[2]. P.K.Paul and M.K.Ghose, "Cloud computing: possibilities, challenges and opportunities with special reference to its emerging need in the academic and working area of Information Science," In Procedia Engineering, vol. (23), pp.2222-2227, Jan 2012.

[3]. Kandukuri, Balachandra Reddy, and Atanu Rakshit, "Cloud security issues,” InServices Computing, 2009. SCC\&\#39;09. IEEE International Conference on IEEE, pp. 517-520, Sep 2009.

[4]. L. Adleman, "Molecular computation of solutions to combinational problems". American Association for the Advancement of Science, pp.1021-1024, 1994.

[5]. Rachna.A, and Anshu.P, "Maintaining Data Confidentiality and Security over Cloud: An Overview", International Journal of Engineering Research and Applications (IJERA), Vol. (4), pp.1922-1926, July 2013.

[6]. D.Sureshraj and Dr.V.Murali Bhaskaran, Automatic DNA Sequence Generation for Secured Effective Multi-Cloud Storage, Journal of Computer Engineering (IOSR-JCE), vol.15, pp. 86-94, Nov-Dec 2013.

[7]. W. Nam, P. Doll'ar, and J. H. Han, "Local Decorrelation For Improved Detection," Nips, pp. 1-9, 2014.

[8]. P. Doll'ar, S. Belongie, and P. Perona, "The Fastest Pedestrian Detector in the West," in Procedings of the British Machine Vision Conference 2010. British Machine Vision Association, 2010, pp. 68.1-68.11 